\title{
Covered stents versus Bare-metal stents in chronic atherosclerotic Gastrointestinal Ischemia (CoBaGl): study protocol for a randomized controlled trial
}

Louisa J. D. van Dijk ${ }^{1,2^{*}}$ (D, Jihan Harki', Desirée van Noord ${ }^{1,3}$, Hence J. M. Verhagen ${ }^{4}$, Jeroen J. Kolkman ${ }^{5,6}$, Robert H. Geelkerken ${ }^{7,8}$, Marco J. Bruno ${ }^{1 \dagger}$, Adriaan Moelker ${ }^{2 \dagger}$ and on behalf of the Dutch Mesenteric Ischemia Study group (DMIS)

\begin{abstract}
Background: Chronic mesenteric ischemia (CMI) is the result of insufficient blood supply to the gastrointestinal tract and is caused by atherosclerotic stenosis of one or more mesenteric arteries in $>90 \%$ of cases. Revascularization therapy is indicated in patients with a diagnosis of atherosclerotic CMI to relieve symptoms and to prevent acute-onchronic mesenteric ischemia, which is associated with high morbidity and mortality. Endovascular therapy has rapidly evolved and has replaced surgery as the first choice of treatment in CMI. Bare-metal stents (BMS) are standard care currently, although retrospective studies suggested significantly higher patency rates for covered stents (CS). The Covered stents versus Bare-metal stents in chronic atherosclerotic Gastrointestinal Ischemia (CoBaGl) trial is designed to prospectively assess the patency of CS versus BMS in patients with atherosclerotic CMI.
\end{abstract}

Methods/design: The CoBaGl trial is a randomized controlled, parallel-group, patient- and investigator-blinded, superiority, multicenter trial conducted in six centers of the Dutch Mesenteric Ischemia Study group (DMIS). Eighty-four patients with a consensus diagnosis of atherosclerotic CMI are 1:1 randomized to either a balloon-expandable BMS (Palmaz Blue with rapid-exchange delivery system, Cordis Corporation, Bridgewater, NJ, USA) or a balloon-expandable CS (Advanta V12 over-the-wire, Atrium Maquet Getinge Group, Hudson, NH, USA). The primary endpoint is the primary stent-patency rate at 24 months assessed with $C T$ angiography. Secondary endpoints are primary stent patency at 6 and 12 months and secondary patency rates, freedom from restenosis, freedom from symptom recurrence, freedom from re-intervention, quality of life according the EQ-5D-5 L and SF-36 and cost-effectiveness at 6, 12 and 24 months.

Discussion: The CoBaGl trial is designed to assess the patency rates of CS versus BMS in patients treated for CMI caused by atherosclerotic mesenteric stenosis. Furthermore, the CoBaGl trial should provide insights in the quality of life of these patients before and after stenting and its cost-effectiveness. The CoBaGl trial is the first randomized controlled trial performed in CMl caused by atherosclerotic mesenteric artery stenosis.

Trial registration: ClinicalTrials.gov, ID: NCT02428582. Registered on 29 April 2015.

Keywords: Endovascular revascularization, Bare-metal stent, Covered stent, Chronic mesenteric ischemia, Atherosclerosis, Celiac artery, Superior mesenteric artery

\footnotetext{
* Correspondence: I.vandijk@erasmusmc.nl

${ }^{\dagger}$ Marco J. Bruno and Adriaan Moelker contributed equally to this work.

'Department of Gastroenterology and Hepatology, Erasmus MC University

Medical Center, 's-Gravendijkwal 230, 3015 CE Rotterdam, The Netherlands

2Department of Radiology, Erasmus MC University Medical Center,

's-Gravendijkwal 230, 3015 CE Rotterdam, The Netherlands

Full list of author information is available at the end of the article
}

(C) The Author(s). 2019 Open Access This article is distributed under the terms of the Creative Commons Attribution 4.0 International License (http://creativecommons.org/licenses/by/4.0/), which permits unrestricted use, distribution, and reproduction in any medium, provided you give appropriate credit to the original author(s) and the source, provide a link to the Creative Commons license, and indicate if changes were made. The Creative Commons Public Domain Dedication waiver (http://creativecommons.org/publicdomain/zero/1.0/) applies to the data made available in this article, unless otherwise stated. 


\section{Background}

Chronic mesenteric ischemia (CMI) is the result of insufficient blood supply to the gastrointestinal (GI) tract and is caused by atherosclerotic stenosis of one or more mesenteric arteries in $>90 \%$ of cases $[1,2]$. The mesenteric arteries are the celiac artery (CA), superior mesenteric artery (SMA) and inferior mesenteric artery (IMA). Classic symptoms of CMI are postprandial abdominal pain and weight loss due to fear of eating. However, CMI may present atypically with constant abdominal discomfort, nausea, vomiting, diarrhea or even constipation [1]. The "classic triad" of CMI consisting of postprandial abdominal pain, weight loss and abdominal bruit is present in only $16-22 \%$ of patients $[3,4]$.

The diagnosis of CMI is established by consensus in a multidisciplinary meeting joined by vascular surgeons, gastroenterologists and interventional radiologists [1]. Consensus is an accepted method of diagnosing if a gold standard test is absent [5]. Symptoms alone do not predict the diagnosis of CMI accurately [4, 6, 7]. Therefore, consensus diagnosis is based on the combination of clinical symptoms, radiological evaluation of the mesenteric vasculature and, if available, assessment of mucosal ischemia with a functional test such as gastricjejunal tonometry $[3,8,9]$ or visible light spectroscopy (VLS) $[10,11]$. A definitive diagnosis of CMI is established if successful therapy of patients with CMI consensus diagnosis results in a durable relief of presenting symptoms.

Revascularization therapy is indicated in patients with a diagnosis of atherosclerotic CMI to relieve symptoms and to prevent acute-on-chronic mesenteric ischemia, which is associated with high morbidity and mortality. Endovascular revascularization has largely replaced open surgical revascularization [1, 12, 13]. Endovascular revascularization of the mesenteric arteries is achieved by means of stent placement. The SMA and CA are target vessels for therapy because of their larger diameter compared to the IMA. Furthermore, a protective collateral network ensures blood supply when the IMA is occluded as shown in patients with an aortic stent occluding the IMA. Literature on revascularization of the IMA is scarce. A study reported successful stenting of the IMA in four patients who were not candidates for CA or SMA revascularization [14]. The classic percutaneous approach for mesenteric endovascular procedure is trans-brachial and trans-femoral, but a trans-radial approach is currently gaining popularity [15].

Endovascular revascularization is associated with a significant decreased risk of in-hospital complications compared to open mesenteric surgical revascularization [16]. Despite the advantages of endovascular revascularization short term, restenosis is a common event occurring in $28-55 \%$ of patients within 2 years after endovascular mesenteric stenting $[13,17]$ whereas $0-25 \%$ of surgically treated patients develop restenosis $[2,18]$. The primary patency rate of open surgical revascularization is significantly higher than that of endovascular revascularization (cumulative odds ratio (OR) 3.57, 95\% confidence interval (CI) 1.82-6.87, $p=0.0002$, [19].

Currently, balloon-expendable bare-metal stents (BMS) are standard care for mesenteric endovascular revascularization $[1,20]$. Retrospective data, however, showed significantly higher primary patency rates of balloon-expendable covered stents (CS) at 3 years for mesenteric artery stenosis [21]. Furthermore, patients treated with CS had less restenosis, symptom recurrence and re-intervention than patients treated with BMS [21]. The Covered versus Balloon Expandable Stent Trial (COBEST) showed significantly higher patency rates of CS than BMS for aortoiliac arterial disease 18, 24, 48 and 60 months after stent placement [22, 23]. Possibly, the membrane that covers the vascular atherosclerotic lesion functions as a physical barrier for intimal hyperplasia and is, therefore, associated with less restenosis in contrast with BMS. The performance of CS for mesenteric artery stenosis is promising, but prospective confirmation is lacking. The CoBaGI trial is a multicenter, randomized controlled, patient- and investigator-blinded clinical trial designed to compare patency rates of the CS versus standard care therapy with BMS in patients with CMI based on atherosclerosis.

\section{Study hypothesis}

CS have a significantly higher patency rate than BMS in patients with CMI due to stenosis of the CA and/or SMA of atherosclerotic origin.

\section{Methods/design}

This study protocol is written according to the Standard Protocol Items: Recommendations for Interventional Trials (SPIRIT) 2013 Statement for study protocols of clinical trials [24]. The SPIRIT Diagram is shown as Table 1 and the SPIRIT Checklist is added in Additional file 1.

\section{Study design}

The CoBaGI trial is a randomized controlled, parallelgroup, patient- and investigator-blinded, superiority, multicenter trial conducted in six centers of the Dutch Mesenteric Ischemia Study group (DMIS). Patients with atherosclerotic CMI will be randomly allocated to standard care therapy using a BMS (Palmaz Blue with rapid-exchange delivery system, Cordis Corporation, Bridgewater, NJ, USA) or a CS (Advanta V12 overthe-wire, Atrium Maquet Getinge Group, Hudson, $\mathrm{NH}$, USA). 
Table 1 Schedule of enrollment, interventions and assessments for the $\mathrm{CoBaGl}$ trial according to Standard Protocol Items: Recommendations for Interventional Trials (SPIRIT) [24]

\begin{tabular}{|c|c|c|c|c|c|c|c|}
\hline \multirow[b]{3}{*}{ Time point } & \multicolumn{7}{|c|}{ Study period } \\
\hline & \multirow{2}{*}{$\frac{\text { Enrollment }}{-t_{1}}$} & \multirow{2}{*}{$\begin{array}{l}\text { Allocation } \\
0\end{array}$} & \multicolumn{4}{|c|}{ Post allocation } & \multirow{2}{*}{$\frac{\text { Close-out }}{t_{x}}$} \\
\hline & & & $t_{1}$ & $t_{2}$ & $t_{3}$ & $t_{4}$ & \\
\hline \multicolumn{8}{|l|}{ Enrollment: } \\
\hline Eligibility screen & $x$ & & & & & & \\
\hline Informed consent & $x$ & & & & & & \\
\hline Allocation & & $x$ & & & & & \\
\hline \multicolumn{8}{|l|}{ Interventions: } \\
\hline Intervention CS & & & $x$ & & & & \\
\hline Intervention BMS & & & $x$ & & & & \\
\hline \multicolumn{8}{|l|}{ Assessments: } \\
\hline CTA & $x$ & & & $x$ & $x$ & $x$ & \\
\hline EQ-5D-5 L & $x$ & & & $x$ & $x$ & $x$ & \\
\hline SF-36 & $x$ & & & $x$ & $x$ & $x$ & \\
\hline Cost-effectiveness & $x$ & & & $x$ & $x$ & $x$ & \\
\hline SAE & & & & $x$ & $x$ & $x$ & $x$ \\
\hline Mortality & & & & $x$ & $x$ & $x$ & $x$ \\
\hline
\end{tabular}

$-t_{1}=$ screening and enrollment, $t_{1}=$ endovascular-stent placement, $t_{2}=6$ months, $t_{3}=12$ months, $t_{4}=24$ months after intervention, $t_{x}=$ end of follow-up at 24 months

BMS bare-metal stent, CS covered stent, CTA computed tomography angiography, SAE serious adverse event, SF-36 36-item Short Form Health Survey

\section{Study setting}

The CoBaGI trial is conducted within the framework of the DMIS. Six Dutch centers participate in the CoBaGI trial. The initiating center is Erasmus MC University Medical Center, an academic hospital in Rotterdam together with Medisch Spectrum Twente in Enschede which serves as a CMI-specialized referral center. Furthermore, four other Dutch tertiary referral centers participate in the study: Maasstad Hospital in Rotterdam; St. Antonius Hospital in Nieuwegein; Bernhoven Hospital in Uden, and Jeroen Bosch Hospital in 'sHertogenbosch.

\section{Eligibility criteria}

Patients with a consensus diagnosis of atherosclerotic CMI eligible for endovascular-stent placement will be included in this trial.

Inclusion criteria are:

- A consensus diagnosis of CMI based on atherosclerotic mesenteric artery stenosis at the origin of the CA and/or SMA, established in a multidisciplinary meeting attended by a gastroenterologist, interventional radiologist and vascular surgeon. This consensus diagnosis is based on:
- Typical symptoms (presence of postprandial pain, unexplained weight loss)

- Significant stenosis of $>50 \%$ of at least one of the mesenteric arteries on previous computed tomography angiography (CTA)*

- Functional assessment of mucosal ischemia with VLS or tonometry

- $\quad$ Aged $\geq 18$ years old

- Signed informed consent

- Total length of mesenteric stenosis $<25 \mathrm{~mm}$

"Requirements for CTA are: CTA performed within the year before consensus diagnosis maximum slice thickness $1 \mathrm{~mm}$, enhancement in aorta $300 \mathrm{HU}$.

Exclusion criteria are:

- Absence of informed consent

- Aged $<18$ years old

- No stenosis detected during angiography

- Renal insufficiency (glomerular filtration rate (GFR) below $30 \mathrm{ml} / \mathrm{min}$ or GFR below $60 \mathrm{ml} / \mathrm{min}$ in the presence of comorbidities relevant to renal function)

- Previous stent placement in the target vessel

- Pregnancy

- Stenosis based on median arcuate ligament syndrome (MALS)

- Stenosis based on vasculitis

- Other criteria which the physician considers incompatible with inclusion in this trial

\section{Recruitment and consent}

Patients with CMI based on atherosclerotic mesenteric artery stenosis are eligible for the $\mathrm{CoBaGI}$ trial. A gastroenterologist, surgeon or research physician will screen the patient according to the inclusion and exclusion criteria. When the patient is eligible for study inclusion, the treating physician will inform the patient about the possibility of participating in the CoBaGI trial and will hand out the patient information form (Additional file 2). Furthermore, the treating physician will inform the patient that study participation is voluntary and that participation may be withdrawn at any time without the need of having to provide a reason. At the return visit, the patient is given the opportunity to ask questions. If the patient consents to participate in the CoBaGI trial, written informed consent is obtained.

\section{Randomization}

Patients are 1:1 randomized for a BMS or CS with a web-based randomization module (9 Knots Business Solutions Ltd., Mansfield, UK) in random blocks of four or six stratified by center. 


\section{Interventions}

At the start of the endovascular procedure patients are randomized for either a BMS or CS. The approach of the intervention is at the discretion of the attending interventionalist (femoral, brachial, radial or retrograde). During the angiography the stenoses are assessed and if a suspected stenosis is not significant, this vessel will not be treated. If the stenosis is detected to be significant during angiography and the stenosis length is $<25 \mathrm{~mm}$ the stenosis will be treated with the allocated stent. In case multiple stenosis are present and eligible for inclusion (i.e., CA and SMA), the same allocated stent type is used. After completion of the intervention various procedural details, such as duration, approach, stent length, treated vessels and complications, are entered in webbased case record forms by the interventionalist. The antiplatelet regimen in the CoBaGI trial consists of aspirin and clopidogrel combined for 12 months followed by aspirin lifelong and applies for both treatment arms. Table 1 presents the schedule of enrollment, interventions and assessments for the CoBaGI trial and Fig. 1 presents the Consolidated Standards of Reporting Trials (CONSORT) study flowchart [25].

\section{Follow-up}

After the endovascular procedure, the patients are followed up at 6,12 and 24 months with CTA to assess stent patency and with a visit to the outpatient clinic to assess symptoms and bodyweight. The patients also receive questionnaires to assess quality of life and costeffectiveness before stent placement and 6, 12 and 24 months after stent placement.

\section{Outcome}

The primary endpoint is the primary patency rate 24 months after stent placement based on intention-to-treat analyses.

The secondary endpoints are (definitions are shown in Table 2):

- Primary patency rates at 6 and 12 months

- Secondary patency rates at 6,12 and 24 months

- Freedom from restenosis at 6, 12 and 24 months

- Freedom from symptom recurrence at 6, 12 and 24 months

- Freedom from re-intervention at 6,12 and 24 months

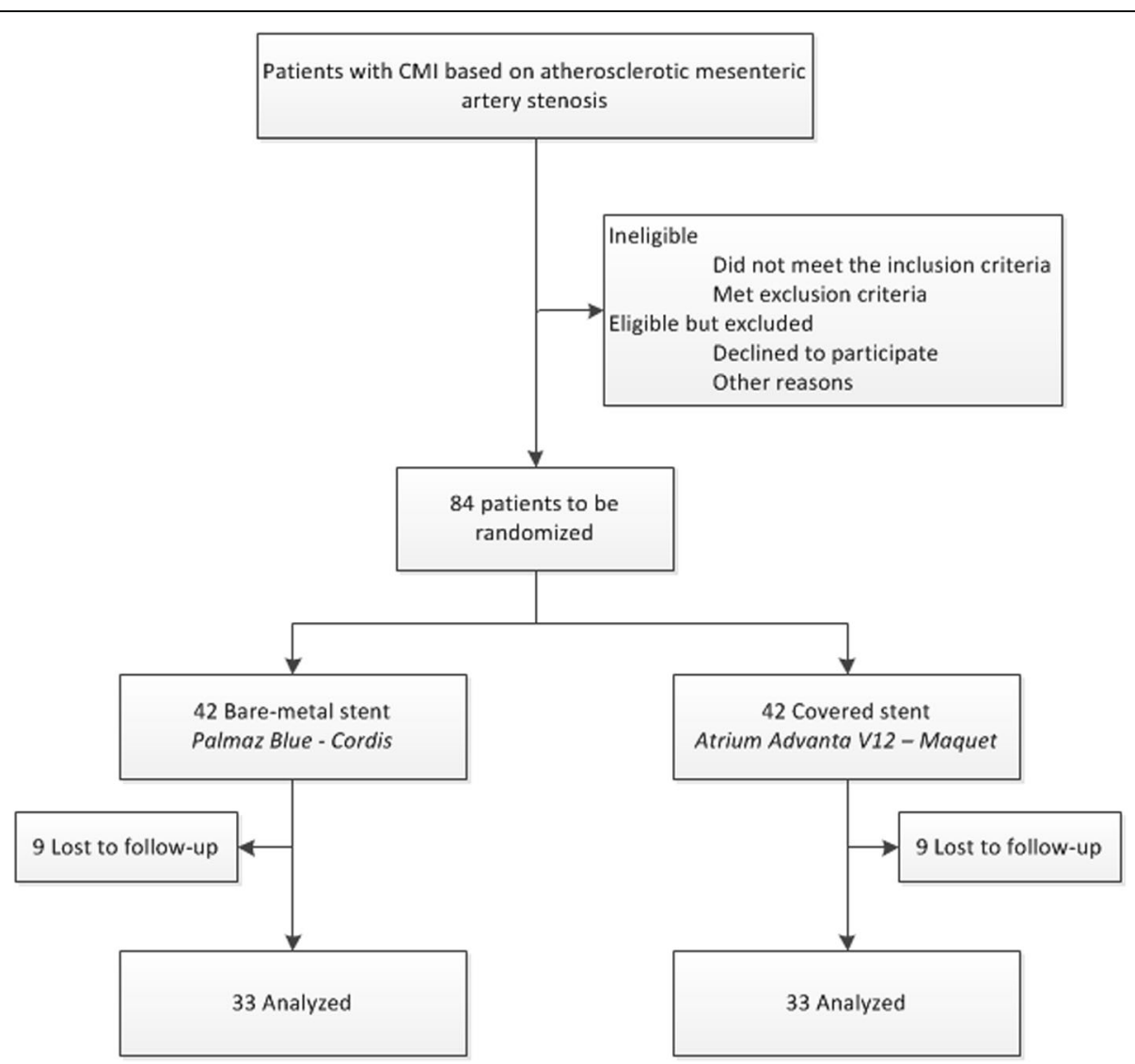

Fig. 1 Flowchart of participants in the Covered stents versus Bare-metal stents in chronic atherosclerotic Gastrointestinal Ischemia (CoBaGl) trial according to Consolidated Standards of Reporting Trials (CONSORT) [25] 
Table 2 Definitions

\begin{tabular}{ll}
\hline & Definition \\
\hline Restenosis & $>50 \%$ intra-stent stenosis regardless whether the patient had clinical symptoms \\
Symptom recurrence & Occurrence of presenting symptoms regardless of stent patency \\
Re-intervention & Intervention due to symptom occurrence in the presence of $>50 \%$ intra-stent \\
& stenosis, either a reimplantation of stent, or a surgical procedure \\
\hline
\end{tabular}

- Quality of life at 6,12 and 24 months

- Cost-effectiveness at 6,12 and 24 months

\section{Blinding}

The patient, investigator and the treating physician at the outpatient clinic are blinded for the treatment allocation (BMS or CS). For the duration of the trial the allocated stent will not be disclosed unless there is a medical need (i.e., restenosis, stent fracture).

\section{Sample size}

The sample size calculation for this randomized controlled, superiority trial is based on the retrospective data of Oderich et al. [21]. Assuming that CS improves the patency rate of BMS after 24 months from $63 \%$ to $95 \%$, and accounting for a $20 \%$ dropout rate, 42 participants are required per arm to achieve $80 \%$ power with a two-sided alpha of 0.05 . The sample size calculation is performed using the Comparison of Independent Proportions Test (SAS Power and Sample size, SAS Institute Inc., Cary, NC, USA).

\section{Data collection methods}

Clinical data are collected using web-based case record forms (9 Knots Business Solutions Ltd., Mansfield, UK). Quality of life is assessed by the validated EuroQol fivedimension, five-level questionnaire (EQ-5D-5 L) $[26,27]$ and 36-item Short-Form Health Survey (SF-36) [28, 29] questionnaires. The cost-effectiveness questionnaire deals with work omission, hospital visits and use of healthcare. All trial documents are kept for 15 years after study completion.

\section{Data management}

Data entry is performed by local study personnel or personnel of the initiating study center. Data entry is anonymized replacing all patient names with a code. Only these codes are used as reference in reports and publications about this investigation. The web-based case record forms contain range checks for data values. The handling of personal data is compliant with the Dutch Personal Data Protection Act, the Code of Good Behavior and the General Data Protection Regulation effective since May 2018.

\section{Data monitoring}

Institution of a data monitoring committee (DMC) was waived by the Ethical Committee because the added risk of the interventional study arm is considered to be negligible. An interim analysis will not be performed. The initiating center, Erasmus MC University Medical Center, is responsible for the data monitoring activities to ensure data quality.

\section{Statistical methods}

Descriptive analyses will be provided regarding patient characteristics before randomization. Because this is a randomized controlled trial, statistical tests to detect differences in these baseline characteristics between both intervention arms will not be performed. The following patient characteristics before randomization will be provided: age, gender, Body Mass Index (BMI), smoking status, comorbidity with hypertension, dyslipidemia, diabetes and cardiovascular disease. Furthermore presenting symptoms will be described as abdominal pain, postprandial pain, exercise-related pain, nausea, diarrhea, weight loss and symptom duration. Patient characteristics will be described as numbers and percentages for dichotomous variables, or as means and standard deviations for continuous variables with normal distribution or medians and interquartile ranges (IQRs) for continuous variables if not normally distributed.

The primary analysis will be performed according the intention-to-treat principle. A per-protocol analysis will also be performed for the primary outcome of stent patency for those patients who were treated according to the treatment protocol.

The primary endpoint of stent patency will be analyzed with a "two-sample z-test" for comparison of two proportions. Furthermore, a predictive model for time-to-event will be obtained by proportional hazards regression and Kaplan-Meier analysis to compare patency rates of CS versus BMS. All other continuous outcome measures will be reported as difference in mean improvement between the CS versus the BMS group. Change in quality of life assessed by the validated questionnaires EQ-5D-5 L [26, 27] and SF-36 $[28,29]$ will be compared between the CS and BMS groups. Furthermore, the costs of both groups will be compared. All costs will be estimated based on actual input in terms of resource use, personnel and indirect 
costs from loss of productivity assessed by the costeffectiveness questionnaire.

\section{Harms}

Adverse events are defined as any undesirable experience occurring to a subject during the study, whether or not considered related to the implantation of a BMS or CS. A serious adverse event (SAE) is any untoward medical occurrence or effect that at any dose:

- Results in death

- Is life threatening (at the time of the event)

- Requires hospitalization or prolongation of existing inpatients' hospitalization

- Results in persistent or significant disability or incapacity

- Is a congenital anomaly or birth defect

- Any other important medical event that may not result in death, be life threatening or require hospitalization, may be considered a serious adverse experience when, based upon appropriate medical judgment, the event may jeopardize the subject and may require an intervention to prevent one of the outcomes listed above

All physicians who are involved in the trial are asked to report all adverse events to the coordinating investigator. The coordinating investigator will report the SAEs through the web portal ToetsingOnline (https://www. toetsingonline.nl) of the Dutch Central Committee on Research involving human subjects to the accredited Institutional Review Board that approved the protocol, within 15 days after the coordinating investigator has first knowledge of the serious adverse reactions. Serious adverse events that result in death or are life threatening should be reported expedited. The expedited reporting will occur not later than 7 days after the responsible investigator has first knowledge of the adverse reaction. This is for a preliminary report with another 8 days for completion of the report.

\section{Auditing}

Representatives of the initiating center, Erasmus MC University Medical Center, audit the participating centers during the course of the study. All protocol modifications are communicated to the relevant parties.

\section{Research ethics approval}

The CoBaGI trial is performed in accordance with declaration of Helsinki and the Dutch law regarding research involving human subjects (Wet Medisch wetenschappelijk Onderzoek met mensen (WMO)). The Institutional Review Board of Erasmus MC University Medical Center, Rotterdam, the Netherlands approved the study protocol on 15 October 2013. The boards of the other five Dutch participating centers gave permission for conducting the trial in their center. The CoBaGI trial was registered on 29 April, 2015 at ClinicalTrials.gov with identification number NCT02428582.

\section{Protocol amendments}

Five protocol amendments were approved after initial approval of the $\mathrm{CoBaGI}$ protocol by the Institutional Review Board. The subjects of these amendments were the addition of new inclusion centers, the addition of one inclusion criterion and adjustment of the patient information form. The contents of these amendments have been incorporated into this protocol.

\section{Confidentially}

The investigators and the study staff will keep all information about the study patients during and after the trial in strict confidence. All study data are saved in the web-based case record forms. This study database is anonymized, the names of the enrolled study patients are replaced with a patient study number.

\section{Ancillary and post-trial care}

Compensation for post-trial care, for those patients who suffered harm from trial participation, is covered by trial health insurance.

\section{Dissemination policy}

The study protocol and study results will be presented at scientific conferences and in peer-reviewed publications. Authorship eligibility follows the common standards of author responsibility, conflict of interest, transparency and the recommendations of the International Committee of Medical Journal Editors (http://www.icmje.org). There are no limitations or restrictions for publication.

\section{Discussion}

The CoBaGI trial is designed to compare the patency rate of BMS (standard care) with CS in patients with CMI based on atherosclerotic mesenteric stenosis. Besides, the study should provide insights into the change in quality of life of patients induced by both stents and their cost-effectiveness. The CoBaGI trial is the first randomized controlled trial to compare BMS with CS in patients with CMI based on an atherosclerotic mesenteric stenosis.

The recently published clinical practice guidelines of the European Society of Vascular Surgery (ESVS) recommend with a low level of evidence (C: expert opinion and/or small studies, retrospective studies) to consider CS over BMS for patients requiring mesenteric artery stenting based a single retrospective study by Oderich et al. [21]. A stronger, level-A recommendation can only be 
issued based on the results of randomized controlled trials. The optimal design would be a double-blinded controlled trial. Since the physician who performs the endovascular procedure cannot be blinded, the CoBaGI trial is designed as a patient- and investigator-blinded, randomized controlled trial.

The primary endpoint of the CoBaGI trial is primary stent patency assessed with CTA. The primary endpoint is a hard endpoint which can be assessed objectively. The secondary endpoint of symptom recurrence is dependent on reports of patient and investigator. In order to ensure unbiased collection and an objective assessment of the endpoints, blinding of the patient and investigator is important.

To perform a fair comparison between the two stent designs all participating centers will use the same brand/ type of BMS as standard of care in the CoBaGI trial. The initiating center, Erasmus MC University Medical Center, had already ample experience with the BMS Palmaz Blue from Cordis for mesenteric stenting and, therefore, this stent was chosen to be used in all participating centers. BMS from different manufacturers are considered comparable in use and outcome although studies comparing BMS from different manufacturers are lacking.

Of note is the inclusion criterion that the length of the stenosis cannot exceed $24 \mathrm{~mm}$ because all stenoses needing stent extension had to be excluded in the CoBaGI trial since a CS will obstruct blood flow when a sidebranch is over-stented in contrast to a BMS. The longest available Palmaz Blue BMS during the inclusion of the CoBaGI trial had a length of $24 \mathrm{~mm}$ and the longest CS had a length of $59 \mathrm{~mm}$.

A pivotal inclusion criterion for the CoBaGI trial is a consensus diagnosis of CMI based on atherosclerotic mesenteric artery stenosis which is established in a multidisciplinary meeting joined by gastroenterologists, interventional radiologists and vascular surgeons. Medisch Spectrum Twente and Erasmus MC University Medical Center are specialized CMI referral centers. All patients eligible for the CoBaGI trial, therefore, will be discussed during a CMI multidisciplinary meeting in one of these two centers. If functional assessment of mucosal ischemia is required to reach a consensus diagnosis, especially in case of single-vessel disease, the patient is referred to Erasmus MC University Medical Center (VLS) $[10,11]$ or Medisch Spectrum Twente (24-h tonometry) [3, 8, 9].

After endovascular revascularization for CMI, antiplatelet therapy is recommended and dual antiplatelet therapy might be considered for 3-12 months [1]. Exact dose and duration schedules are lacking for mesenteric revascularization. All stented patients in the $\mathrm{CoBaGI}$ trial, regardless of allocation, will receive aspirin and clopidogrel for 12 months after stenting followed by aspirin lifelong.
The true incidence of atherosclerotic CMI is unknown. It is expected that in the upcoming years the incidence of atherosclerotic CMI will increase, mainly because of the aging population and the increasing prevalence of cardiovascular disease. The predicted increase in the prevalence of atherosclerotic CMI, the significant patient burden in terms of pain and loss of quality of life, and, in particular, the risk of an acute ischemic event with an exceedingly high mortality risk, underscore the importance of the CoBaGI trial. The CoBaGI trial aimed to improve the patient outcome in terms of pain relief, improvement of quality of life and prevention of acute ischemic events. The fact that the CoBaGI trial is carried out within the framework of the DMIS with patients being recruited from multiple academic and community hospitals should facilitate the generalizability of the study outcomes.

In conclusion, the CoBaGI trial is a multicenter, randomized controlled, patient- and investigator-blinded superiority trial comparing BMS (standard of care) with CS in patients with CMI caused by an atherosclerotic stenosis at the origin of the mesenteric artery.

\section{Trial status}

The CoBaGI trial started inclusion in May 2015. In September 2018, 71 patients of the 84 (85\%) patients had been included. Inclusion is expected to be completed at the end of 2018. Follow-up duration is 2 years. Latest protocol version is version 5, date 28 June 2017.

\section{Additional files}

Additional file 1: Standard Protocol Items: Recommendations for Interventional Trials (SPIRIT) 2013 Checklist: recommended items to address in a clinical trial protocol and related documents*. (DOC $121 \mathrm{~kb}$ )

Additional file 2: Translated patient information form of the CoBaGl trial. (DOCX $46 \mathrm{~kb}$ )

\section{Abbreviations}

BMI: Body Mass Index; BMS: Bare-metal stent(s); CA: Celiac artery; CMI: Chronic mesenteric ischemia; CS: Covered stents; CTA: Computed tomography angiography; DMC: Data Monitoring Committee; DMIS: Dutch Mesenteric Ischemia Study group; GFR: Glomerular filtration rate; GI: Gastrointestinal; IMA: Inferior mesenteric artery; IQR: Interquartile range; MALS: Median arcuate ligament syndrome; SAE: Serious adverse event; SMA: Superior mesenteric artery; VLS: Visible light spectroscopy; WMO: Wet Medisch wetenschappelijk Onderzoek met mensen (Dutch law regarding research involving human subjects)

\section{Acknowledgements}

The authors wish to acknowledge bio-statistician Bettina E Hansen for her help with the sample size and statistical analysis plan.

Independent physicians (all in the Netherlands and in alphabetical order) Bob J van Wely, Department of Surgery, Bernhoven Hospital in Uden; Annick C Weustink, Department of Radiology, Erasmus MC University Medical Center in Rotterdam; Jochim S Terhaar sive Droste, Department of Gastroenterology and Hepatology, Jeroen Bosch Hospital in 's-Hertogenbosch; Jan-Hein J Hensen, Department of Radiology, Maasstad Hospital in Rotterdam; Harold W Hom, Department of Intensive Care, Medisch Spectrum Twente in Enschede; 
Hieronymus H Vincent, Department of Internal Medicine, St. Antonius Hospital in Nieuwegein.

Clinical centers and principal investigators (all in the Netherlands and in alphabetical order)

Bernhoven Hospital in Uden: André S van Petersen; Erasmus MC University Medical Center in Rotterdam: Adriaan Moelker; Jeroen Bosch Hospital in 's-Hertogenbosch: Jan Willem Hinnen; Maasstad Hospital in Rotterdam: Dammis Vroegindeweij; Medisch Spectrum Twente in Enschede: Robert H Geelkerken; St. Antonius Hospital in Nieuwegein: Daniel AD van den Heuvel. The Dutch Mesenteric Ischemia Study group (DMIS) consists of (all in the Netherlands and in alphabetical order):

Ron Balm, Academic Medical Centre, Amsterdam

Gert Jan de Borst, University Medical Centre Utrecht, Utrecht Juliette T Blauw, Medisch Spectrum Twente, Enschede

Marco J Bruno, Erasmus MC University Medical Center, Rotterdam

Olaf J Bakker, St. Antonius Hospital, Nieuwegein

Louisa JD van Dijk, Erasmus MC University Medical Center, Rotterdam

Hessel CJL Buscher, Gelre Hospitals, Apeldoorn

Bram Fioole, Maasstad Hospital, Rotterdam

Robert H Geelkerken, Medisch Spectrum Twente, Enschede

Jaap F Hamming, Leiden University Medical Center, Leiden

Jihan Harki, Erasmus MC University Medical Center, Rotterdam

Daniel AF van den Heuvel, St. Antonius Hospital, Nieuwegein

Eline S van Hattum, University Medical Centre Utrecht, Utrecht

Jan Willem Hinnen, Jeroen Bosch Hospital, 's-Hertogenbosch

Jeroen J Kolkman, Medisch Spectrum Twente, Enschede

Maarten J van der Laan, University Medical Center Groningen, Groningen

Kaatje Lenaerts, Maastricht University Medical Center, Maastricht

Adriaan Moelker, Erasmus MC University Medical Center, Rotterdam

Desirée van Noord, Franciscus Gasthuis and Vlietland, Rotterdam

Maikel P Peppelenbosch, Erasmus MC University Medical Center, Rotterdam

André S van Petersen, Bernhoven Hospital, Uden

Pepijn Rijnja, Medisch Spectrum Twente, Enschede

Peter J van der Schaar, St. Antonius Hospital, Nieuwegein

Luke G Terlouw, Erasmus MC University Medical Center, Rotterdam

Hence JM Verhagen, Erasmus MC University Medical Center, Rotterdam

Jean Paul PM de Vries, University Medical Center Groningen, Groningen

Dammis Vroegindeweij, Maasstad Hospital, Rotterdam

\section{Access to data}

All investigators will have access to the final trial dataset.

\section{Authors' contributions}

LVD and JH drafted the manuscript. DVN, MB, HV, JK, RG and AM coauthored the writing of the manuscript. JH, DvN, MB, HV, JK, RG and AM participated in the design of the study. AM is the principal investigator. All authors critically revised the manuscript, read and approved the final manuscript.

\section{Funding}

An unrestricted grant was received from Atrium Maquet Getinge Group. The $\mathrm{CoBaGl}$ trial is an investigator-initiated trial. The sponsor had no influence on the design of the study, data collection, results or publications.

\section{Availability of data and materials}

The datasets used and/or analyzed during the current study are available from the corresponding author on reasonable request.

\section{Ethics approval and consent to participate}

The CoBaGl trial is performed in accordance with the Declaration of Helsinki and the Dutch law regarding research involving human subjects (Wet Medisch wetenschappelijk Onderzoek met mensen (WMO)). The Institutional Review Board of Erasmus MC University Medical Center, Rotterdam, the Netherlands approved the study protocol with identification number MEC-2013-476 on 15 October 2013. The boards of the other five Dutch participating centers gave permission for conducting the trial in their center: Maasstad Hospital in Rotterdam on 12 November 2015, Medisch Spectrum Twente in Enschede on 9 June 2016, St. Antonius Hospital in Nieuwegein on 14 September 2016, Bernhoven Hospital in Uden on 28 November 2016 and Jeroen Bosch Hospital in 's-Hertogenbosch on 23 November 2017. Informed consent will be obtained from all study participants.

\section{Consent for publication}

Not applicable.

\section{Competing interests}

LVD: no competing interests. JH: no competing interests. DVN: no competing interests. HV: no competing interests. JK: no competing interests. RG: no competing interests. MB: no competing interests. AM: no competing interests.

\begin{abstract}
Author details
'Department of Gastroenterology and Hepatology, Erasmus MC University Medical Center, 's-Gravendijkwal 230, 3015 CE Rotterdam, The Netherlands. ${ }^{2}$ Department of Radiology, Erasmus MC University Medical Center, 's-Gravendijkwal 230, 3015 CE Rotterdam, The Netherlands. ${ }^{3}$ Department of Gastroenterology and Hepatology, Franciscus Gasthuis and Vlietland, Kleiweg 500, 3045 PM Rotterdam, The Netherlands. "Department of Vascular Surgery, Erasmus MC University Medical Center, 's-Gravendijkwal 230, 3015 CE Rotterdam, The Netherlands. ${ }^{5}$ Department of Gastroenterology and Hepatology, Medisch Spectrum Twente, Postbus 50 000, 7500 KA Enschede, The Netherlands. 'Department of Gastroenterology and Hepatology, University Medical Center Groningen, Postbus 30.001, 9700 RB Groningen, The Netherlands. ${ }^{7}$ Department of Vascular Surgery, Medisch Spectrum Twente, Postbus 50 000, 7500 KA Enschede, The Netherlands. ${ }^{8}$ TechMed Centre, Faculty Science and Technology, University Twente, Postbus 50000 7500 KA Enschede, The Netherlands.
\end{abstract}

Received: 21 October 2018 Accepted: 23 July 2019

Published online: 20 August 2019

\section{References}

1. Bjorck M, et al. Editor's choice-Management of the diseases of mesenteric arteries and veins: clinical practice guidelines of the European Society of Vascular Surgery (ESVS). Eur J Vasc Endovasc Surg. 2017;53(4):460-510.

2. Clair DG, Beach JM. Mesenteric ischemia. N Engl J Med. 2016;374(10):959-68.

3. Mensink PB, et al. Clinical significance of splanchnic artery stenosis. Br J Surg. 2006;93(11):1377-82.

4. Sana A, et al. Radiological imaging and gastrointestinal tonometry add value in diagnosis of chronic gastrointestinal ischemia. Clin Gastroenterol Hepatol. 2011;9(3):234-41.

5. Rutjes AW, et al. Evaluation of diagnostic tests when there is no gold standard. A review of methods. Health Technol Assess. 2007;11(50):iii-x 51.

6. ter Steege RW, et al. Splanchnic artery stenosis and abdominal complaints: clinical history is of limited value in detection of gastrointestinal ischemia. World J Surg. 2012;36(4):793-9.

7. Mensink PB, Moons LM, Kuipers EJ. Chronic gastrointestinal ischaemia: shifting paradigms. Gut. 2011;60(5):722-37.

8. Mensink PB, et al. Twenty-four hour tonometry in patients suspected of chronic gastrointestinal ischemia. Dig Dis Sci. 2008;53(1):133-9.

9. Otte JA, et al. Clinical impact of gastric exercise tonometry on diagnosis and management of chronic gastrointestinal ischemia. Clin Gastroenterol Hepatol. 2005;3(7):660-6.

10. Van Noord D, et al. Endoscopic visible light spectroscopy: a new, minimally invasive technique to diagnose chronic Gl ischemia. Gastrointest Endosc. 2011;73(2):291-8.

11. Friedland $S$, et al. Diagnosis of chronic mesenteric ischemia by visible light spectroscopy during endoscopy. Gastrointest Endosc. 2007;65(2):294-300.

12. Fidelman N, et al. ACR Appropriateness Criteria ${ }^{\oplus}$ radiologic management of mesenteric ischemia. J Am Coll Radiol. 2017;14(5s):S266-71.

13. Pillai $A K$, et al. Quality improvement guidelines for mesenteric angioplasty and stent placement for the treatment of chronic mesenteric ischemia. J Vasc Interv Radiol. 2018;29(5):642-7.

14. Wohlauer $\mathrm{M}$, et al. Inferior mesenteric artery stenting as a novel treatment for chronic mesenteric ischemia in patients with an occluded superior mesenteric artery and celiac trunk. Eur J Vasc Endovasc Surg. 2014;27(3):e21-3.

15. Fischman AM, Swinburne NC, Patel RS. A technical guide describing the use of transradial access technique for endovascular interventions. Tech Vasc Interv Radiol. 2015;18(2):58-65.

16. Alahdab F, et al. A systematic review and meta-analysis of endovascular versus open surgical revascularization for chronic mesenteric ischemia. J Vasc Surg. 2018;67(5):1598-605. 
17. Bulut $\mathrm{T}$, et al. Long-term results of endovascular treatment of atherosclerotic stenoses or occlusions of the coeliac and superior mesenteric artery in patients with mesenteric ischaemia. Eur J Vasc Endovasc Surg. 2017;53(4):583-90.

18. Oderich GS, Gloviczki P, Bower TC. Open surgical treatment for chronic mesenteric ischemia in the endovascular era: when it is necessary and what is the preferred technique? Semin Vasc Surg. 2010;23(1):36-46.

19. Saedon $\mathrm{M}$, et al. Endovascular versus surgical revascularization for the management of chronic mesenteric ischemia. Vasc Endovasc Surg. 2015; 49(1-2):37-44.

20. Blauw JT, et al. Mesenteric vascular treatment 2016: from open surgical repair to endovascular revascularization. Best Pract Res Clin Gastroenterol. 2017;31(1):75-84

21. Oderich GS, et al. Comparison of covered stents versus bare metal stents for treatment of chronic atherosclerotic mesenteric arterial disease. J Vasc Surg. 2013:58(5):1316-23.

22. Mwipatayi BP, et al. Durability of the balloon-expandable covered versus bare-metal stents in the Covered versus Balloon Expandable Stent Trial. (COBEST) for the treatment of aortoiliac occlusive disease. J Vasc Surg. 2016; 64(1):83-94.e1.

23. Mwipatayi BP, et al. A comparison of covered vs bare expandable stents for the treatment of aortoiliac occlusive disease. J Vasc Surg. 201 1;54(6):1561-70.

24. Chan AW, et al. SPIRIT 2013 Statement: defining standard protocol items for clinical trials. Ann Intern Med. 2013;158(3):200-7.

25. Moher D, et al. CONSORT 2010 explanation and elaboration: updated guidelines for reporting parallel group randomised trials. Bmj. 2010;340:c869.

26. Janssen MF, et al. Measurement properties of the EQ-5D-5L compared to the EQ-5D-3L across eight patient groups: a multi-country study. Qual Life Res. 2013;22(7):1717-27.

27. Herdman $\mathrm{M}$, et al. Development and preliminary testing of the new fivelevel version of EQ-5D (EQ-5D-5L). Qual Life Res. 2011;20(10):1727-36.

28. Aaronson NK, et al. Translation, validation, and norming of the Dutch language version of the SF-36 Health Survey in community and chronic disease populations. J Clin Epidemiol. 1998:51(11):1055-68.

29. Ware JE Jr, Sherbourne CD. The MOS 36-item short-form health survey (SF-36). I. Conceptual framework and item selection. Med Care. 1992;30(6):473-83.

\section{Publisher's Note}

Springer Nature remains neutral with regard to jurisdictional claims in published maps and institutional affiliations.

Ready to submit your research? Choose BMC and benefit from:

- fast, convenient online submission

- thorough peer review by experienced researchers in your field

- rapid publication on acceptance

- support for research data, including large and complex data types

- gold Open Access which fosters wider collaboration and increased citations

- maximum visibility for your research: over $100 \mathrm{M}$ website views per year

At $\mathrm{BMC}$, research is always in progress.

Learn more biomedcentral.com/submissions 